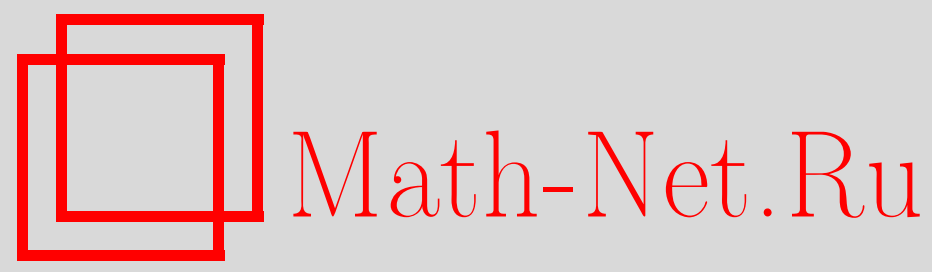

Л. И. Сазонов, Двумерные операторы Тёплица с измеримыми символами, Матем. заметки, 2003, том 74, выпуск 1, 88-98

DOI: https://doi.org/10.4213/mzm248

Использование Общероссийского математического портала Math-Net.Ru подразумевает, что вы прочитали и согласны с пользовательским соглашением http://www . mathnet.ru/rus/agreement

Параметры загрузки:

IP : 3.80 .253 .173

26 апреля 2023 г., 15:41:01

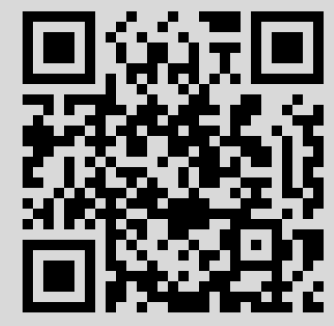




\title{
ДВУМЕРНЫЕ ОПЕРАТОРЫ ТЁПЛИЦА С ИЗМЕРИМЫМИ СИМВОЛАМИ
}

\section{Л. И. Сазонов}

\begin{abstract}
Для полной алгебры двумерных операторов Тёплица с измеримыми ограниченными символами установлены необходимые условия фредгольмовости и результаты о разделении особенностей в символах. В качестве следствий получены достаточные условия фредгольмовости для символов, удовлетворяющих условиям типа локальной секториальности.

Библиография: 11 названий.
\end{abstract}

1. Пусть $\Gamma=\{z ;|z|=1\}$ - единичная окружность в комплексной плоскости $\mathbb{C} ; P$ проектор Рисса в пространстве $L_{p}(\Gamma)(1<p<\infty)$, связанный с оператором сингулярного интегрирования

$$
(S f)(t)=\frac{1}{\pi i} \int_{\Gamma} \frac{f(\tau)}{\tau-t} d \tau
$$

формулой $P=\frac{1}{2}(I+S) ; H_{p}(\Gamma)=P L_{p}(\Gamma)$ - пространство Харди.

Обозначим через $T O_{p}$ банахову подалгебру в алгебре End $H_{p}(\Gamma)$ всех линейных ограниченных операторов, действующих в пространстве $H_{p}(\Gamma)$, порожденную всеми операторами Тёплища $T(a)=P a I$ с символами $a \in L_{\infty}(\Gamma)$. Отметим, что алгебра $T O_{p}$ содержит идеал $\mathcal{K}_{p}$ всех компактных операторов из End $H_{p}(\Gamma)$. Аналогично для пространств Харди $H_{p}(\Gamma \times \Gamma)=(P \otimes P) L_{p}(\Gamma \times \Gamma)$ введем банахову алгебру $T O_{p}^{(2)}$, порожденную всеми двумерными операторами Тёплица $T^{(2)}(a)=(P \otimes P) a I$ с символами $a \in L_{\infty}(\Gamma \times \Gamma)$.

Алгебра $T O_{p}^{(2)}$ содержит идеал всех компактных операторов $\mathcal{K}_{p}^{0}=\mathcal{K}_{p} \widehat{\otimes} \mathcal{K}_{p}$ и банахову подалгебру $T O_{p} \widehat{\otimes} T O_{p}$ с замкнутыми двусторонними идеалами $\mathcal{K}_{p}^{1}=\mathcal{K}_{p} \widehat{\otimes} O_{p}$, $\mathcal{K}_{p}^{2}=T O_{p} \widehat{\otimes} \mathcal{K}_{p}$. (Здесь через $\mathcal{A} \widehat{\otimes} \mathcal{B}$, где $\mathcal{A}, \mathcal{B}$ - банаховы подалгебры в End $H_{p}(\Gamma)$, обозначается банахова подалгебра в End $H_{p}(\Gamma \times \Gamma)$, являющаяся замьканием множества всех операторов вида $\sum A_{i} \otimes B_{i}$, где $A_{i} \in \mathcal{A}, B_{i} \in \mathcal{B}$.)

В исследованиях фредгольмовости двумерных операторов Тёплища (см. [1] и приведенную там библиографию) рассматривались различные классы операторов Тёплища из алгебры $T O_{p} \widehat{\otimes} T O_{p}$. При этом в некоторых случаях применялась схема билокализации, впервые предложенная в [2]. Ее основные положения применительно к алгебре 
$T O_{p} \widehat{\otimes} T O_{p}$ можно кратко сформулировать следующим образом: ввиду соотношений $\mathcal{K}_{p}^{1} \cdot \mathcal{K}_{p}^{2} \subset \mathcal{K}_{p}^{0}$ обратимость фактор-класса $[A]_{0}=A+\mathcal{K}_{p}^{0}$ оператора $A$ из $T O_{p} \widehat{\otimes} T O_{p}$ эквивалентна одновременной обратимости фактор-классов $[A]_{i}=A+\mathcal{K}_{p}^{i}$ в фактор-алгебрах $T O_{p} \widehat{\otimes} T O_{p} / \mathcal{K}_{p}^{i} \quad(i=1,2) ;$ в свою очередь, обратимость в фактор-алгебрах $T O_{p} \widehat{\otimes}$ $T O_{p} / \mathcal{K}_{p}^{i}$ исследуется с помощю локальных методов; обратимость фактор-класса $[A]_{0}$ в фактор-алгебре $T O_{p} \widehat{\otimes} T O_{p} / \mathcal{K}_{p}^{0}$ влечет его обратимость в полной алгебре End $H_{p}(\Gamma \times$ $\Gamma) / \mathcal{K}_{p}^{0}$, которая эквивалентна фредгольмовости оператора $A$, но доказательство необходимости этого условия требует дополнительных исследований.

Отметим, что непосредственно применить указанную схему к полной алгебре $T O_{p}^{(2)}$ нельзя ввиду того, что множества $\mathcal{K}_{p}^{i}$ не являются идеалами в этой алгебре. Вьход за рамки тензорного произведения (в данном случае $T O_{p} \widehat{\otimes} T O_{p}$ ) был предложен в [3], [4], где была исследована фредгольмовость характеристических бисингулярных операторов с коэффициентами, имеющими разрьвы вдоль гладких кривых. Применительно к алгебре $T O_{p}^{(2)}$ этот подход состоит в рассмотрении ее наибольшей подалгебры, в которой $\mathcal{K}_{p}^{i}(i=1,2)$ остаются двусторонними идеалами. Такая подалгебра содержит операторы Тёплища с символами, выходящими за рамки тензорного произведения $L_{\infty}(\Gamma) \widehat{\otimes}$ $L_{\infty}(\Gamma)$, но не совпадает с полной алгеброй $T O_{p}^{(2)}$.

Ниже реализуется другая возможность, состоящая в расширении множеств $\mathcal{K}_{p}^{i}(i=$ $1,2)$ до двусторонних идеалов $\mathcal{J}_{p}^{i}(i=1,2)$ в алгебре $T O_{p}^{(2)}$, для которых вьполняется соотношение $\mathcal{J}_{p}^{1} \cdot \mathcal{J}_{p}^{2} \subset \mathcal{K}_{p}^{0}$. Это позволяет применить к алгебре $T O_{p}^{(2)}$ с идеалами $\mathcal{J}_{p}^{1}$ и $\mathcal{J}_{p}^{2}$ схему билокализации.

2. Определим множество $\mathcal{J}_{p}^{i}(i=1,2)$ как замыкание в алгебре $T O_{p}^{(2)}$ множества всех операторов вида $\sum A_{j} K_{j} B_{j}$, где $A_{j}, B_{j} \in T O_{p}^{(2)}, K_{j} \in \mathcal{K}_{p}^{i}$.

Справедлива

Лемма 1. Множества $\mathcal{J}_{p}^{i}(i=1,2)$ являются замкнутыми двусторонними идеалами в алгебре $Т O_{p}^{(2)}$, причем

$$
\mathcal{J}_{p}^{1} \cdot \mathcal{J}_{p}^{2}, \mathcal{J}_{p}^{2} \cdot \mathcal{J}_{p}^{1} \subset \mathcal{K}_{p}^{0}
$$

ДокАЗАТЕЛЬСТво. Очевидно, в проверке нуждается лишь соотношение (1). В свою очередь, для этого достаточно установить компактность операторов вида $\left(K_{1} \otimes I\right) A(I \otimes$ $\left.K_{2}\right),\left(I \otimes K_{2}\right) A\left(K_{1} \otimes I\right)$, где $K_{1}, K_{2}$ - компактные операторы в пространстве $H_{p}(\Gamma)$, а оператор $A$ имеет вид $A=\Pi A_{j}$, где $A_{j}=T^{(2)}\left(a_{j}\right), a_{j} \in L_{\infty}(\Gamma \times \Gamma)$. Не нарушая общности, будем считать, что операторы $K_{j}$ определяются формулами

$$
\left(K_{j} f\right)(t)=\phi_{j}(t) \int_{\Gamma} \psi_{j}(s) f(s) d s
$$

где $\phi_{j}, \psi_{j} \in L_{\infty}(\Gamma)$. В этом случае неравенство

$$
\begin{aligned}
\left\|\left(K_{1} \otimes I\right) f\right\|_{L_{p}\left(\Gamma_{1}, L_{q}\left(\Gamma_{2}\right)\right)} & \leqslant\left\|\phi_{1}\right\|_{L_{p}}\left\|\int_{\Gamma} \psi_{1}(s) f\left(s, t_{2}\right) d s\right\|_{L_{q}\left(\Gamma_{2}\right)} \\
& \leqslant c\left\|\phi_{1}\right\|_{L_{p}}\left\|\psi_{1}\right\|_{L_{\infty}}\|f\|_{L_{q}\left(\Gamma_{2}, L_{r}\left(\Gamma_{1}\right)\right)}
\end{aligned}
$$


устанавливает ограниченность оператора

$$
K_{1} \otimes I: L_{q}\left(\Gamma_{2}, L_{r}\left(\Gamma_{1}\right)\right) \rightarrow L_{p}\left(\Gamma_{1}, L_{q}\left(\Gamma_{2}\right)\right)
$$

при любых $q, r, p$ из $(1, \infty)$. Здесь $\Gamma=\Gamma_{1}=\Gamma_{2}$ снабжено индексами для различения пространств со смешанными нормами.

Аналогично, при любых $p, q, r \in(1, \infty)$ ограничен оператор

$$
I \otimes K_{2}: L_{q}\left(\Gamma_{1}, L_{r}\left(\Gamma_{2}\right)\right) \rightarrow L_{p}\left(\Gamma_{2}, L_{q}\left(\Gamma_{1}\right)\right)
$$

Далее, заметим, что для каждой функции $a \in L_{\infty}(\Gamma \times \Gamma)$ существует последовательность функций $a_{n} \in L_{\infty}(\Gamma) \otimes L_{\infty}(\Gamma)$ такая, что

$$
\left\|a_{n}\right\|_{L_{\infty}} \leqslant\|a\|_{L_{\infty}}, \quad \lim \left\|a_{n}-a\right\|_{L_{p}}=0
$$

при любом $p \in[1, \infty)$.

Действительно, достаточно положить $a_{n}=\left(Q_{n} \otimes Q_{n}\right) a$, где $Q_{n}-$ проектор вида

$$
\left(Q_{n} f\right)(t)=\sum_{j=1}^{n} \frac{n \chi_{j}(t)}{2 \pi} \int_{\Gamma_{j}} f(\tau) d \tau
$$

где $\Gamma=\bigcup_{j=1}^{n} \Gamma_{j}$ - разбиение окружности на $n$ равных дуг, $\chi_{j}-$ характеристическая функция дуги $\Gamma_{j}$. Известно [5, с. 20], что проекторы $Q_{n} \otimes Q_{n}$ сильно сходятся к единичному оператору $I$ при $n \rightarrow \infty$ в любом пространстве $L_{p}(\Gamma \times \Gamma)(1<p<\infty)$.

Пусть $A_{n}=\prod_{j} A_{j, n}$, где $A_{j, n}=T^{(2)}\left(a_{j, n}\right),\left\{a_{j, n}\right\}$ - соответствуюшая последовательность для символа $a_{j}$. Так как оператор $P \otimes P$ ограничен в любом пространстве $L_{p}\left(\Gamma, L_{q}(\Gamma)\right)(1<p, q<\infty)$ (см., например, [6, с. 338]), то имеет место оценка

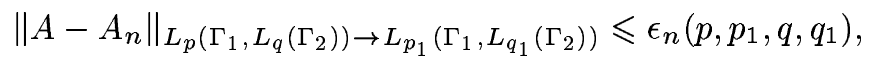

где $\epsilon_{n} \rightarrow 0$ при $n \rightarrow \infty$ для любых фиксированных $p, p_{1}, q, q_{1}$, удовлетворяющих условиям $1<p_{1}<p<\infty, 1<q_{1}<q<\infty$.

Действительно, для одного оператора имеем

$$
\begin{aligned}
\left\|T^{(2)}\left(a-a_{n}\right) f\right\|_{L_{p_{1}}\left(\Gamma_{1}, L_{q_{1}}\left(\Gamma_{2}\right)\right)} & \leqslant c\|\|\left(a-a_{n}\right) f\left\|_{L_{q_{1}}\left(\Gamma_{2}\right)}\right\|_{L_{p_{1}}\left(\Gamma_{1}\right)} \\
& \leqslant c\|\| a-a_{n}\left\|_{L_{\mu}\left(\Gamma_{2}\right)}\right\| f\left\|_{L_{q}\left(\Gamma_{2}\right)}\right\|_{L_{p_{1}}\left(\Gamma_{1}\right)} \\
& \leqslant c\left\|a-a_{n}\right\|_{L_{\nu}\left(\Gamma_{1}, L_{\mu}\left(\Gamma_{2}\right)\right)}\|f\|_{L_{p}\left(\Gamma_{1}, L_{q}\left(\Gamma_{2}\right)\right)} \\
& \leqslant c_{1}\left\|a-a_{n}\right\|_{L_{\sigma}(\Gamma \times \Gamma)}\|f\|_{L_{p}\left(\Gamma_{1}, L_{q}\left(\Gamma_{2}\right)\right)},
\end{aligned}
$$

где $1 / \mu=1 / q_{1}-1 / q, 1 / \nu=1 / p_{1}-1 / p, \sigma=\max (\mu, \nu)$.

Здесь использованы неравенство Гёльдера и непрерьвность вложения

$$
L_{p}\left(\Gamma_{1}, L_{q}\left(\Gamma_{2}\right)\right) \subset L_{p_{1}}\left(\Gamma_{1}, L_{q_{1}}\left(\Gamma_{2}\right)\right)
$$

при $p_{1} \leqslant p, q_{1} \leqslant q$. Для произведения операторов доказательство соотношения $(2)$ аналогично предыдущему. 
После этого получаем

$$
\begin{aligned}
\left\|\left(K_{1} \otimes I\right)\left(A-A_{n}\right)\left(I \otimes K_{2}\right) f\right\|_{L_{p}\left(\Gamma_{1} \times \Gamma_{2}\right)} & \leqslant c_{1}\left\|\left(A-A_{n}\right)\left(I \otimes K_{2}\right) f\right\|_{L_{p}\left(\Gamma_{2}, L_{r}\left(\Gamma_{1}\right)\right)} \\
& \leqslant c_{1} \epsilon_{n}\left\|\left(I \otimes K_{2}\right) f\right\|_{L_{p_{1}}\left(\Gamma_{2}, L_{r_{1}}\left(\Gamma_{1}\right)\right)} \\
& \leqslant c_{2} \epsilon_{n}\|f\|_{L_{r_{1}}\left(\Gamma_{1}, L_{p}\left(\Gamma_{2}\right)\right)}
\end{aligned}
$$

Так как $r$ - любое число из $(1, \infty)$, а $r_{1}$ - любое число, меньшее $r$, можно положить $r_{1}=p$ и, следовательно, справедливо соотношение

$$
\lim _{n \rightarrow \infty}\left\|\left(K_{1} \otimes I\right)\left(A-A_{n}\right)\left(I \otimes K_{2}\right)\right\|_{L_{p}(\Gamma \times \Gamma) \rightarrow L_{p}(\Gamma \times \Gamma)}=0,
$$

влекущее компактность оператора $\left(K_{1} \otimes I\right) A\left(I \otimes K_{2}\right)$, так как компактны операторы $\left(K_{1} \otimes I\right) A_{n}\left(I \otimes K_{2}\right)$. Лемма доказана.

Учитывая, что для функции $a \in C(\Gamma)$ операторы $P a(I-P),(I-P) a P$ компактны в любом пространстве $L_{p}(\Gamma)(1<p<\infty)$, приходим к следуюшему утверждению.

Лемма 2. Пусть $a \in C(\Gamma)$. Тогда для любого оператора $A \in T O_{p}^{(2)}$ справедливь соотношения

$$
[T(a) \otimes I, A] \in \mathcal{J}_{p}^{1}, \quad[I \otimes T(a), A] \in \mathcal{J}_{p}^{2},
$$

әде $[A, B]=A B-B A-$ коммутатор операторов $A$ и $B$.

Идеалы $\mathcal{J}_{p}^{j}$ допускают следующее внутреннее описание.

ЛЕмма 3. Пусть $M_{t}$ - множество всех функиий из $C(\Gamma)$ таких, что каждая из них равна единице в некоторой окрестности точки $t$ и принимает значение в $[0,1]$. Oператор $A \in T O_{p}^{(2)}$ принадлежкит идеалу $\mathcal{J}_{p}^{1}\left(\mathcal{J}_{p}^{2}\right)$ тогда и только тогда, когда для каждой точки $t$ выполняется соотношение

$$
\lim _{n \rightarrow \infty}\left\|\left(b_{n} \otimes I\right) A\right\|=0 \quad\left(\lim _{n \rightarrow \infty}\left\|\left(I \otimes b_{n}\right) A\right\|=0\right),
$$

әде $\left\{b_{n}\right\}$ - любая последовательность функиий из $M_{t}$ такая, что $\operatorname{supp} b_{n}$ стягиваются в точку $t$ при $n \rightarrow \infty$.

ДоказАтельство. Рассмотрим случай идеала $\mathcal{J}_{p}^{1}$.

Необходимость. Достаточно установить вьполнение соотношения (3) для операторов вида

$$
A_{N}=T^{(2)}\left(a_{N}\right) T^{(2)}\left(a_{N-1}\right) \ldots T^{(2)}\left(a_{2}\right) K_{1}, \quad N=1,2, \ldots,
$$

где $K_{1} \in \mathcal{K}_{p}^{1}, a_{j} \in L_{\infty}(\Gamma \times \Gamma)$.

Применим индукцию по $N$. Для оператора $K_{1}(N=1)$ утверждение верно, так как оператор $K_{1}$, как и все операторы из $\mathcal{K}_{p}^{1}$, равномерно аппроксимируется операторами вида $\sum L_{i} \otimes A_{i}$, где $L_{i}$ - компактные операторы, а для любого компактного в $H_{p}(\Gamma)$ оператора $L$ ввиду сильной сходимости операторов $b_{n} I$ к нулю имеем $\lim _{n \rightarrow \infty}\left\|b_{n} L\right\|=0$.

Предположим, что соотношение (3) верно для всех операторов $K_{m-1}$ вида (4) с $N=$ $m-1$. Тогда для функции $b \in M_{t}$ такой, что $b b_{n}=b_{n}$, получаем представление

$$
\begin{aligned}
b_{n} K_{m}= & -\left(\left(b_{n} P b(I-P)\right) \otimes P\right) a_{m} K_{m-1} \\
& +\left(\left(b_{n}(I-P) b P\right) \otimes I\right) K_{m}+\left(\left(b_{n} P\right) \otimes P\right) a_{m} b K_{m-1},
\end{aligned}
$$


из которого вытекает неравенство

$$
\left\|b_{n} K_{m}\right\| \leqslant c\left(\left\|b_{n} P b(I-P)\right\|+\left\|b_{n}(I-P) b P\right\|+\left\|b K_{m-1}\right\|\right)
$$

где константа $c$ не зависит от $b_{n}$ и $b$. По предположению индукции $\left\|b K_{m-1}\right\|$ сколь угодно мало при достаточно малом носителе функции $b$; первые два слагаемых стремятся $\mathrm{k}$ нулю при $n \rightarrow \infty$ вследствие компактности операторов $P b(I-P)$ и $(I-P) b P$.

Таким образом,

$$
\lim _{n \rightarrow \infty}\left\|b_{n} K_{m}\right\|=0
$$

что и доказьвает необходимость.

Достаточность. Ввиду соотношения (3) для любого $\epsilon>0$ сушествует непрерьвное разложение единицы $\left\{b_{\epsilon, j}\right\}$ на $\Gamma$ такое, что

$$
\left\|\left(b_{\epsilon, j} \otimes I\right) A\right\|<\epsilon
$$

и система функций $\left\{b_{\epsilon, j}^{\prime}\right\}$ такая, что $b_{\epsilon, j}^{\prime} \in \bigcup M_{t}, b_{\epsilon, j}^{\prime}$ равна единице на $\operatorname{supp} b_{\epsilon, j}$ и система множеств supp $b_{\epsilon, j}^{\prime}$ имеет кратность пересечения равную двум. Тогда вследствие леммы 2 оператор $A$ с точностью до оператора из идеала $\mathcal{J}_{p}^{1}$ совпадает с оператором $A_{\epsilon}=\sum\left(T\left(b_{\epsilon, j}\right) \otimes I\right) A\left(T\left(b_{\epsilon, j}^{\prime}\right) \otimes I\right)$, для которого аналогично лемме 1.5 из [7] устанавливается оценка $\left\|A_{\epsilon}\right\| \leqslant c \epsilon$, где $c$ не зависит от $\epsilon$. Отсюда в силу замкнутости идеала $\mathcal{J}_{p}^{1}$ имеем $A \in \mathcal{J}_{p}^{1}$, что и завершает доказательство леммы 3.

ЗАмечаниЕ 1 . Множество $\mathcal{K}_{p}^{1}\left(\mathcal{K}_{p}^{2}\right)$ выделяется в $T O_{p}^{(2)}$ следуюшим образом: оператор $A \in T O_{p}^{(2)}$ принадлежит $\mathcal{K}_{p}^{1}\left(\mathcal{K}_{p}^{2}\right)$ тогда и только тогда, когда

$$
\lim _{n \rightarrow \infty}\left\|\left(P_{n} \otimes I\right) A\left(P_{n} \otimes I\right)-A\right\|=0 \quad\left(\lim _{n \rightarrow \infty}\left\|\left(I \otimes P_{n}\right) A\left(I \otimes P_{n}\right)-A\right\|=0\right)
$$

для любой системы конечномерных проекторов $\left\{P_{n}\right\}$ таких, что

$$
s_{n \rightarrow \infty} \lim _{n}=I, \quad s-\lim _{n \rightarrow \infty} P_{n}^{*}=I .
$$

Здесь $s$ - lim означает сильньй предел, а операция *-переход к сопряженному оператору.

3. Оператор $A \in T O_{p}^{(2)}$ будем называть фредгольмовым (частично $i$-фредгольмо$\boldsymbol{в b \iota}(i=1,2))$ в алгебре $T O_{p}^{(2)}$, если его фактор-класс $[A]_{0}\left([A]_{i}\right)$ обратим в фактор-алгебре $T O_{p}^{(2)} / \mathcal{K}_{p}^{0}\left(T O_{p}^{(2)} / \mathcal{J}_{p}^{i}\right)$. Заметим, что это эквивалентно существованию его левого и правого регуляризаторов (частичных $i$-регуляризаторов), т.е. таких операторов $R_{1}$ и $R_{2}$ из $T O_{p}^{(2)}$, что операторы $R_{1} A-I, A R_{2}-I$ принадлежат идеалу $\mathcal{K}_{p}^{0}\left(\mathcal{J}_{p}^{i}\right)$.

Из леммы 1 непосредственно вытекает

Лемма 4. Оператор $A$ фредгольмов в алгебре $T O_{p}^{(2)}$ тогда и только тогда, когда он одновременно частично 1- и 2-фредгольмов в алгебре ТО $\mathrm{p}_{p}^{(2)}$.

Установим некоторые необходимые условия фредгольмовости (частичной фредгольмовости) в алгебре $T O_{p}^{(2)}$.

Предварительно сформулируем некоторые известные факты о вектор- и операторфункциях. 
Пусть $X$ - банахово сепарабельноепространство; $(S, \mu)$-пространство с мерой. Вектор-функиией назьвается отображение $x: S \rightarrow X$, обозначаемое также $\{x(s)\}$ или $\{s \rightarrow x(s)\}$. Вектор-функция назьвается слабо (сильно) измеримой, если для любого функционала $f$ из сопряженного пространства $X^{*}$ числовая функция $f(x(s)) \mu$-измерима (существует последовательность $\mu$-простых вектор-функций $\left\{x_{n}(s)\right\}$ такая, что почти для всех $\left.s \lim _{n \rightarrow \infty}\left\|x(s)-x_{n}(s)\right\|=0\right)$.

Согласно теореме Петтиса [8, с. 187] для сепарабельного пространства $X$ оба типа измеримости совпадают, поэтому будем говорить просто об измеримости. Ясно, что можно рассматривать вектор-функции, которые заданы почти всюду. Банаховы пространства $L_{p}(S, X) \quad(1 \leqslant p \leqslant \infty)$ определяются как множества всех измеримых вектор-функций с конечной нормой

$$
\|x\|_{L_{p}(S, X)}=\left\{\int_{S}\|x(s)\|_{X}^{p} d \mu(s)\right\}^{1 / p}, \quad 1<p<\infty, \quad\|x\|_{L_{\infty}(S, X)}=\operatorname{ess} \sup \|x(s)\| .
$$

Обычные пространства функций $L_{p, q}(S \times T)$ со смешанной нормой

$$
\|x\|=\left\{\int_{S}\left\{\int_{T}|x(s, t)|^{q} d \nu(t)\right\}^{p / q} d \mu(s)\right\}^{1 / p}
$$

отождествляется с пространством вектор-функций $L_{p}\left(S, L_{q}(T)\right)$.

Оператор-функция $\{A(s)\}$, определенная почти для всех $s$ и принимающая значения в End $X$, называется измеримой, если для любого $x \in X$ измерима вектор-функция $\{A(s) x\}$.

Оператор-функции $A: S \rightarrow$ End $X$ сопоставим оператор $M(\{A(s)\})$, назьваемьй оператором умножения на оператор-функцию и определяемый соотношением

$$
(M(\{A(s)\}) f)(s)=\{A(s) f(s)\}
$$

Этот оператор ограничен в любом пространстве $L_{p}(S, X)(1<p<\infty)$ тогда и только тогда, когда $\|A(s)\|$ - сушественно ограниченная функция, и его норма определяется формулой

$$
\|M(\{A(s)\})\|=\|\| A(s)\|\|_{L_{\infty}(S)} .
$$

Множество всех измеримых существенно ограниченных оператор-функций $A: S \rightarrow$ End $X$ образует банахово пространство $L_{\infty}(S$, End $X)$, более того, оно является банаховой алгеброй относительно умножения оператор-функций. Ввиду (5) эта алгебра изометрически изоморфна подалгебрев $\operatorname{End} L_{p}(S, X)$, состоящей из операторов умножения на оператор-функции из $L_{\infty}(S$, End $X)$.

Перечисленные свойства оператор-функций установлены в случае гильбертова пространства в [9], но без труда переносятся на более общий случай сепарабельного банахова пространства.

Лемма 5. Отображсния, определяемые на операторах вида

$$
A=\sum_{i} \prod_{j} T^{(2)}\left(a_{i j}\right), \quad a_{i j} \in L_{\infty}(\Gamma \times \Gamma)
$$


соотношениями

$$
\Phi(A)=\sum_{i} \prod_{j} a_{i j}, \quad \Phi^{1}(A)=\left\{\sum_{i} \prod_{j} T\left(a_{i j}(t, \cdot)\right)\right\}, \quad \Phi^{2}(A)=\left\{\sum_{i} \prod_{j} T\left(a_{i j}(\cdot, t)\right)\right\}
$$

u сопоставляющие оператору соответственно функиию из $L_{\infty}(\Gamma \times \Gamma)$ и оператор-функиии из $L_{\infty}\left(\Gamma, T O_{p}\right)$, продолжаются до непрерывных гомоморфизмов

$$
\Phi: T O_{p}^{(2)} \rightarrow L_{\infty}(\Gamma \times \Gamma), \quad \Phi^{i}: T O_{p}^{(2)} \rightarrow L_{\infty}\left(\Gamma, T O_{p}\right)
$$

ДокАЗАТЕЛЬСТво. Пусть $U$ - оператор в $L_{p}(\Gamma)$ вида $(U f)(t)=t f(t)$. Тогда из соотношения

$$
s-\lim _{n \rightarrow \infty} U^{-n} P U_{n}=I
$$

вытекает справедливость для операторов вида (6) равенств

$$
\begin{aligned}
s-\lim _{n \rightarrow \infty}\left(U^{-n} \otimes U^{-n}\right) A\left(P U^{n} \otimes P U^{n}\right) & =M(\Phi(A)) \\
s-\lim _{n \rightarrow \infty}\left(U^{-n} \otimes I\right) A\left(P U_{n} \otimes I\right) & =M\left(\Phi^{1}(A)\right) \\
s-\lim _{n \rightarrow \infty}\left(I \otimes U^{-n}\right) A\left(I \otimes P U^{n}\right) & =M\left(\Phi^{2}(A)\right)
\end{aligned}
$$

где $M(\Phi(A))$ и $M\left(\Phi^{i}(A)\right)$ - соответственно операторы умножения на функцию $\Phi(A) \in$ $L_{\infty}(\Gamma \times \Gamma)$ и оператор-функцию $\Phi^{i}(A) \in L_{\infty}\left(\Gamma, T O_{p}\right)$.

Из соотношений (7)-(9) следует, что

$$
\|M(\Phi(A))\| \leqslant\|P\|^{2}\|A\|, \quad\left\|M\left(\Phi^{i}(A)\right)\right\| \leqslant\|P\|\|A\| .
$$

Но ввиду изометрической изоморфности алгебры операторов умножения на функции из $L_{\infty}(\Gamma \times \Gamma)\left(\right.$ оператор-функции из $\left.\left.L_{\infty}\left(\Gamma, T O_{p}\right)\right)\right)$ алгебре $L_{\infty}(\Gamma \times \Gamma)\left(L_{\infty}\left(\Gamma, T O_{p}\right)\right)$ имеем

$$
\|\Phi(A)\|_{L_{\infty}(\Gamma \times \Gamma)} \leqslant\|P\|^{2}\|A\|, \quad\left\|\Phi^{i}(A)\right\|_{L_{\infty}\left(\Gamma, T O_{p}\right)} \leqslant\|P\|\|A\| .
$$

Поэтому отображения $\Phi, \Phi^{i}$ продолжаются по непрерьвности на всю алгебру $T O_{p}^{(2)}$.

Следствием леммы 5 является

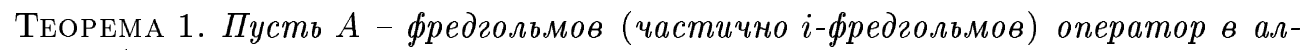
гебре $T O_{p}^{(2)}$. Тогда функиия $\Phi(A)$ (оператор-функиия $\Phi^{i}(A)$ ) обратима в алгебре $L_{\infty}(\Gamma \times \Gamma)\left(L_{\infty}\left(\Gamma, T O_{p}\right)\right)$.

ДоказАтельство.Достаточно заметить, что идеал $\mathcal{K}_{p}^{0}\left(\mathcal{J}_{p}^{i}\right)$ содержится в ядре гомоморфизма $\Phi\left(\Phi^{i}\right)$. В свою очередь, это следует из соотношения $s-\lim _{n \rightarrow \infty} K U^{n}=0$, верного для любого компактного оператора $K$ в пространстве $L_{p}(\Gamma)$.

ЗАмЕчаниЕ 2. Отображения $\Phi, \Phi^{i}$ введены по аналогии с одномерным случаем [1]. 
4. Для исследования частичной $i$-фредгольмовости будем применять к фактор-алгебре $T O_{p}^{(2)} / \mathcal{J}_{p}^{i}$ локальньй принщип Гохберга-Крупника [10], краткая формулировка которого приводится ниже для полноты изложения.

Пусть $\mathfrak{A}$ - банахова алгебра с единицей $е$. Множество ее элементов $M$, не содержашее нуля, назьвается локализующим классом, если

1) $\sup _{a \in M}\|a\|<\infty$

2) для любых $a_{1}, a_{2}$ из $M$ существует $a \in M$ такой, что

$$
a a_{i}=a_{i} a=a, \quad i=1,2 .
$$

Элементы $x, y \in \mathfrak{A}$ называются $M$ - әквивалентными, если

$$
\inf _{a \in M}\|(x-y) a\|=\inf _{a \in M}\|a(x-y)\|=0 .
$$

Элемент $x \in \mathfrak{A}$ называется $M$-обратимым слева (справа), если существуют такие элементы $z \in \mathfrak{A}, a \in M$, что

$$
z x a=a \quad(a x z=a) .
$$

Элемент $x$ называется $M$-обратимым, если он одновременно $M$-обратим слева и справа.

Система локализующих классов $\left\{M_{\tau}\right\}_{\tau \in T}$ назьвается покрывающей, если из любого семейства элементов $\left\{a_{\tau}\right\}_{\tau \in T}\left(a_{\tau} \in M_{\tau}\right)$ можно выбрать конечное число элементов $a_{\tau_{j}}, j=1,2, \ldots, N$, таких, что элемент $\sum a_{\tau_{j}}$ обратим в алгебре $\mathfrak{A}$.

ТЕОРема 2. Пусть $\left\{x_{\tau}\right\}_{\tau \in T}-$ семейство локальных представителей әлемента $x \in \mathfrak{A}$, m.е. для каждого $\tau \in T$ әлементы $x$ и $x_{\tau} M_{\tau}$-эквивалентны. Если элемент $x$ коммутирует со всеми әлементами из $\bigcup_{\tau \in T} M_{\tau}$, то $x$ обратим в алгебре $\mathfrak{A}$ тогда и только тогда, когда для любого $\tau \in T$ әлемент $x_{\tau} M_{\tau}$-обратим.

Для фактор-алгебры $T O_{p}^{(2)} / \mathcal{J}_{p}^{1}$ справедлива

Лемма 6. Пусть $M_{t}^{1}$ - мнохсество всех фактор-классов вида $[T(a) \otimes I]_{1}$, дее $a \in M_{t}$. Тогда $\left\{M_{t}^{1}\right\}_{t \in \Gamma}-$ покрывающая система локализующих классов в алгебре $T O_{p}^{(2)} / \mathcal{J}_{p}^{1}$, причем каждый класс принадлежст иентру алгебры $T O_{p}^{(2)} / \mathcal{J}_{p}^{1}$.

ДокАЗАТЕльство. Принадлежность каждого класса $M_{t}^{1}$ центру алгебры следует из определения идеала $\mathcal{J}_{p}^{1}$ и компактности операторов $P a(I-P),(I-P) a P$ в пространстве $L_{p}(\Gamma)$ для символа $a \in C(\Gamma)$.

Далее, установим неравенство

$$
1 \leqslant \inf _{[A]_{1} \in M_{t}^{1}}\left\|[A]_{1}\right\| \leqslant \sup _{[A]_{1} \in M_{t}^{1}}\left\|[A]_{1}\right\| \leqslant c_{p},
$$

где $c_{p}$ - норма проектора Рисса $P$ в пространстве $L_{p}(\Gamma)$.

Пусть $K_{\epsilon} \in \mathcal{J}_{p}^{1}$ - такой оператор, что

$$
\left\|[A]_{1}\right\|+\epsilon \geqslant\left\|A+K_{\epsilon}\right\| .
$$

Пусть $B$ - оператор умножения на функцию $b$ из $M_{t}$; тогда вследствие леммы 3 найдется такая функция $b$, что

$$
\left\|A+K_{\epsilon}\right\| \geqslant\left\|B\left(A+K_{\epsilon}\right)\right\| \geqslant\|b a\|_{L_{\infty}}-\|b(I-P) a P\|-\left\|B K_{\epsilon}\right\| \geqslant 1-\epsilon .
$$

Таким образом, справедлива левая часть неравенства (10); его правая часть очевидна.

Теперь заметим, что система классов $\left\{M_{t}\right\}_{t \in \Gamma}$ образует покрьвающую систему локализующих классов в алгебре $C(\Gamma)$ и, следовательно, $\left\{M_{t}^{1}\right\}_{t \in \Gamma}$ - покрьвающая система локализующих классов в алгебре $T O_{p}^{(2)} / \mathcal{J}_{p}^{1}$. 
ЗАмЕчАниЕ 3. Аналогичным образом определяется покрывающая система локализующих классов $\left\{M_{t}^{2}\right\}_{t \in T}$ в фактор-алгебре $T O_{p}^{(2)} / \mathcal{J}_{p}^{2}$.

Для операторов Тёплица справедлива следующая теорема о разделении особенностей.

ТЕорема 3. Пусть $a, b \in L_{\infty}(\Gamma \times \Gamma)$ и операторы $T^{(2)}(a)$ и $T^{(2)}(b)$ частично 1-фредгольмовы в алгебре $Т O_{p}^{(2)}$. Если существуют замкнутые непересекающиеся мнохества $F$ и $G$ из $\Gamma$ такие, что $a \in C(\Gamma \backslash F \times \Gamma), b \in C(\Gamma \backslash G \times \Gamma)$, mо оператор $T^{(2)}(a b)$ также является частично 1-фредгольмовым в алгебре тO $O_{p}^{(2)}$.

ДокАЗАТЕЛЬСТво. Для любой точки $\xi \in \Gamma$ одна из функций $a$ или $b$ непрерьвна в некоторой окрестности кривой $\xi \times \Gamma$. Пусть это будет функция $a$. Тогда оператор $T^{(2)}(a b) \quad M_{\xi}^{1}$-эквивалентен оператору $T^{(2)}\left(a_{\xi} b\right)$, где $a_{\xi}(t, \tau)=a(\xi, \tau)$, т.е. $a_{\xi}-$ непрерьвная функция одного переменного $\tau$. $M_{\xi}^{1}$-обратимость оператора $T^{(2)}\left(a_{\xi}\right)$ есть обратимость одномерного оператора Тёплица $T\left(a_{\xi}\right)$ в алгебре $T O_{p}$.

Действительно, после применения к соотношению

$$
R T^{(2)}\left(a_{\xi}\right)(T(a) \otimes I)=(T(a) \otimes I)+K
$$

где $K \in \mathcal{J}_{p}^{1}, a \in M_{\xi}$, означающую левую $M_{\xi}^{1}$-обратимость фактор-класса $\left[T^{(2)}\left(a_{\xi}\right)\right]_{1}$, гомоморфизма $\Phi^{1}$ имеем для почти всех $t$

$$
\Phi^{1}(R)(t) \Phi^{1}\left(T^{(2)}\left(a_{\xi}\right)\right)(t) a(t) I=a(t) I .
$$

Остается заметить, что $\Phi^{1}\left(T^{(2)}\left(a_{\xi}\right)\right)(t)=T\left(a_{\xi}\right)$, поэтому из $(11)$ следует левая обратимость $T\left(a_{\xi}\right)$.

Аналогично устанавливается правая обратимость этого оператора в алгебре $T O_{p}$.

Из результатов об обратимости одномерных операторов Тёплица с непрерьвньми символами [11] следует, что для любого $\epsilon>0$ функция $a_{\xi}$ допускает факторизацию

$$
a_{\xi}=a_{\xi}^{+} d a_{\xi}^{-}
$$

где

$$
\|1-d\|<\epsilon, \quad\left(a_{\xi}^{+}\right)^{ \pm 1} \in C_{+}(\Gamma), \quad\left(a_{\xi}^{-}\right)^{ \pm} \in C_{-}(\Gamma) .
$$

Вследствие этого оператор $T^{(2)}\left(a_{\xi} b\right)$ может быть представлен в виде

$$
T^{(2)}\left(a_{\xi} b\right)=\left(I \otimes T\left(a_{\xi}^{-}\right)\right) T^{(2)}(b d)\left(I \otimes T\left(a_{\xi}^{+}\right)\right) .
$$

Но операторы $I \otimes T\left(a^{ \pm}\right)$обратимы в алгебре $T O_{p}^{(2)}$, а оператор $T^{(2)}(b d)$ при достаточно малом $\epsilon$ частично 1-фредгольмов в алгебре $T O_{p}^{(2)}$.

СЛЕДСТВИЕ 1. Пусть $a, b \in L_{\infty}(\Gamma \times \Gamma)$ и существуют замкнутые множества $F_{1}, G_{1}, F_{2}, G_{2}$ из $\Gamma$ такие, что $F_{i} \cap G_{i}=\oslash, i=1,2, u a \in C\left((\Gamma \times \Gamma) \backslash\left(F_{1} \times F_{2}\right)\right)$, $b \in C\left((\Gamma \times \Gamma) \backslash\left(G_{1} \times G_{2}\right)\right)$. Eсли операторы $T^{(2)}(a)$ и $T^{(2)}(b)$ фредгольмовы в алгебре TO $\mathrm{O}_{p}^{(2)}$, mо оператор $T^{(2)}(a b)$ также фредгольмов в этой алгебре.

Утверждение очевидным образом вытекает из теоремы 3 , ее аналога для частичной 2-фредгольмовости и леммы 4. 
СлЕДСТВИЕ 2. Пусть $b \in C(\Gamma \times \Gamma), \quad a \in L_{\infty}(\Gamma \times \Gamma) u T^{(2)}(a)-\oint p e д г о л ь м о в$ оператор в алгебре $T O_{p}^{(2)}$. Oператор $T^{(2)}(a b)$ фредгольмов в алгебре $T O_{p}^{(2)}$ тогда и только тогда, когда $b \neq 0$ на $Г \times \Gamma$ и имеет нулевые частные индексы.

ДокАЗАТЕЛЬСТво. Достаточность очевидна ввиду следствия 1 и критерия фредгольмовости для двумерных операторов Тёплица с непрерьвньми символами.

Необходимость вытекает из теоремы 1. Применение гомоморфизма $\Phi$ дает обратимость функции $b$ в $L_{\infty}(\Gamma \times \Gamma)$, т.е. выполнение неравенства $b \neq 0$ на $\Gamma \times \Gamma$. Далее, если $b=b_{0} t^{\kappa} \tau^{\mu}$, где $b_{0}$ имеет нулевые частные индексы, то оператор $T^{(2)}\left(b_{0}\right)$ фредгольмов ввиду достаточности, а оператор $T^{(2)}(a b)$, например в случае $\kappa \geqslant 0, \mu \geqslant 0$, представим в виде

$$
T^{(2)}(a b)=T^{(2)}\left(a b_{0}\right) T^{(2)}\left(t^{\kappa} \tau^{\mu}\right)
$$

и применение гомоморфизмов $\Phi^{i}$ приводит к равенствам $\kappa=\mu=0$.

Введем класс символов $S_{p}(\Gamma \times \Gamma)$, к которому отнесем все функции из $L_{\infty}(\Gamma \times \Gamma)$, удовлетворяюшие следующему условию:

для любой точки $(t, \tau) \in \Gamma \times \Gamma$ существует ее окрестность $U$ и константа $c \in \mathbb{C}$ такие, Что

$$
\operatorname{ess}_{\sup }\left|\chi_{U}-c a\right|_{U} \mid<\epsilon_{p}
$$

где $\chi_{U}$ - характеристическая функция множества $U$, а $\epsilon_{p}=\left(\|P\|_{L_{p} \rightarrow L_{p}}\right)^{-2}$.

Лемма 7. Функиия $a \in L_{\infty}(\Gamma \times \Gamma)$ содержится в классе $S_{p}(\Gamma \times \Gamma)$ тогда и только тогда, когда ее мохно представить в виде $a=b s$, где $b \in C(\Gamma \times \Gamma), b \neq 0$ на $Г \times \Gamma$, а s удовлетворяет условию

$$
\operatorname{ess} \sup |1-s|<\epsilon_{p}
$$

ДокАЗАТЕЛьство. Достаточность очевидна. Для доказательства необходимости выберем конечное покрытие $\Gamma \times \Gamma$ указанными окрестностями $U_{\alpha}$ с соответствующими константами $c_{\alpha}$, и построим разложение единицы $\left\{\chi_{\alpha}\right\}$, отвечающие этому покрытию и состоящее из непрерьвных функций.

Имеем оценку

$$
\operatorname{ess} \sup \left|1-\sum c_{\alpha} \chi_{\alpha} a\right| \leqslant \sum \operatorname{ess} \sup \left|\chi_{\alpha}\left(1-a c_{\alpha}\right)\right|<\epsilon_{p} .
$$

Ясно, что функция $\sum c_{\alpha} \chi_{\alpha} \neq 0$ на $\Gamma \times \Gamma$. Поэтому, полагая $b=\left(\sum c_{\alpha} \chi_{\alpha}\right)^{-1}$, получаем нужное представление для $a \mathrm{c} s=a \sum c_{\alpha} \chi_{\alpha}$.

ЗАмЕчаниЕ 4 . В случае $p=2$ класс $S_{p}(\Gamma \times \Gamma)$ совпадает с классом локально секториальных функций [1], т.е. для каждой точки из $\Gamma \times \Gamma$ существует ее окрестность, константа $c(|c|=1)$ и число $\epsilon>0$ такие, что для почти всех точек из этой окрестности $\operatorname{Re}(c a) \geqslant \epsilon$.

Для функции $a \in S_{p}(\Gamma \times \Gamma)$ введем частные индексы, полагая $\operatorname{ind}_{i} a=\operatorname{ind}_{i} b$, где $a=b s$-представление, указанное в предыдущей лемме. Ясно, что эти числа не зависят от этого представления и определяются функцией $a$ однозначно.

Следствие 2 приводит к следующему результату. 
Теорема 4. Пусть $a \in S_{p}(\Gamma \times \Gamma)$. Оператор $T^{(2)}(a)$ является фредгольмовым оператором в алгебре $T O_{p}^{(2)}$ тогда и только тогда, когда $\operatorname{ind}_{i} a=0 \quad(i=1,2)$.

ДокАЗАТЕльСтво. Достаточно заметить, что оператор $T^{(2)}(s)$ обратим в алгебре $T O_{p}^{(2)}$, так как для него справедлива оценка

$$
\left\|I-T^{(2)}(s)\right\|=\left\|T^{(2)}(1-s)\right\|<1 .
$$

\section{СПИСОК ЦИТИРОВАННОЙ ЛИТЕРАТУРЫ}

[1] Bötcher A., Silbermann B. Analysis of Toeplitz operators. Berlin: Akademie-Verlag, 1989.

[2] Пилиди В. С. О многомерных бисингулярных операторах // Докл. АН СССР. 1971. Т. 201. C. $787-789$.

[3] Сазонов Л. И. Бисингулярные характеристические операторы с разрывньми коэффициентами в пространстве $L_{2}\left(R^{2}\right) / /$ Функцион. анализ и его прилож. 1985. Т. 12. № 2. С. $90-91$.

[4] Сазонов Л. И. О бисингулярных операторах с измеримыми коэффициентами // Сиб. матем. ж. 1996. Т. 37. № 2. С. 389-398.

[5] Красносельский М. А., Забрейко П. П., Пустыльник Е. И., Соболевский П. Е. Интегральные операторы в пространствах суммируемых функций. М.: Наука, 1966.

[6] Данфорд Н., Шварц Дж. Т. Линейные операторы. Спектральная теория. М.: Мир, 1966.

[7] Симоненко И. Б. Новый общий метод исследования линейных операторных уравнений типа сингулярных интегральных уравнений // Изв. АН СССР. Сер. матем. 1965. Т. 29. №3. C. $567-586$.

[8] Иосида К. Функциональный анализ. М.: Мир, 1967.

[9] Наймарк М. Ю. Нормированные кольа. М.: Наука, 1968.

[10] Гохберг И. Ц., Крупник Н. Я. Введение в теорию одномерных сингулярных интегральных операторов. Кишинев: Штиинца, 1973.

[11] Гохберг И. Ц., Фельдман И. А. Уравнения в свертках и проекционные методы их решения. М.: Наука, 1971. 